\title{
Quelques réflexions sur le vibrofonçage
}

\section{H. GONIN}

Simecsol

42 , villa Brimborion

92190 Meudon

L'auteur examine dans cet article la facon dont l'énergie est utilisée pour la pénétration d'un élément linéaire dans le sol par vibrofonçage. Pour cela, le sol est supposé être un milieu dans lequel les forces résistantes sont proportionnelles à la vitesse particulaire le long du fût et dont la réaction en pointe obéit à une loi rigide-plastique, le seuil de plasticité étant la « résistance dynamique en pointe i) $q$. La valeur moyenne de la vitesse de pénétration est alors explicitée en fonction des paramètres définissant le vibreur et le sol pour l'onde incidente. En conclusion, l'auteur envisage de quelle manière des essais sur site pourraient permettre d'évaluer directement des caractéristiques définissant les sols dans lesquels on envisage de foncer des éléments et pose la question de savoir si les renseignements ainsi recueillis pourraient être corrélés avec la portance des éléments mis en place.

\section{Some remarks on vibratory driving}

In this paper, the author looks into the use of energy to sink a drill-string into a soil by vibratory driving. The soil is supposed to be a medium where the shaft resistance is in direct ratio to the material velocity and where the tip resistance follows a rigidplastic law. The plastic limit is the idynamic tip stress $x \mathrm{q}$. The value of the mean penetration velocity is expressed in relation with the parameters describing the vibratory device and the soil. for the incident stress-wave, In conclusion, the author examines how to determine by in situ testing some characteristics defining a ground where elements have to be driven, and the fitness of these data to be correlated with the bearing capacity of the driven elements is questioned. 
Heureux l'ingénieur de bureau d'études qu'un client exigeant contraint à sortir de la routine et à engager une réflexion personnelle ! Cela m'est arrivé en 1978 pour les fondations de l'appontement pétrolier de Dunkerque-Gravelines, et, depuis lors, je n'ai cessé d'être passionné par l'étude du battage des pieux, et mon activité professionnelle m'a parfoís donné l'occasion d'enrichissantes applications pratiques.

Mais il n'est pas nécessaire d'être stimulé par l'étude et la réalisation d'ouvrage pour continuer à s'intéresser aux problèmes techniques : ayant maintenant cessé mon activité professionnelle, je garde dans ce domaine quelques hobbies I C'est la raison pour laquelle je présente ici quelques réflexions sur le vibrofonçage, dans la continuité des articles que j'ai consacrés au battage : il y a, en effet, certainement beaucoup à tirer de la comparaison de ces deux méthodes, souvent en concurrence pour le fonçage dans le sol d'éléments linéaires métalliques (pieux, tubes, palplanches).

1

\section{Notations et rappels}

Lorsqu'une force apparait instantanêment au sommet d'une tige élastique supposée infiniment lonque, de section $A$ et dont la matière a une masse volumique $\rho$ et un module d'Young $E$, il nait une onde de compression (ou de traction) de célérité $c=(E / \rho)^{1 / 2}$ (voir figure 1). Le sommet de la tige se déplace à la vitesse $v_{\text {. }}$ (vitesse particulaire) du fait de la compression (ou de la traction) de la tige. Le travail de la force f pendant un temps dt, fv dt, sert à comprimer (ou à étirer) une longueur cdt de la tige, et à mettre en mouvement cette masse comprimée (ou étirée) ( $p$ Acdt). La conservation de l'énergie permet d'écrire que le travail de la force f est égal à la somme de l'énergie de compression et de l'énergie cinétique :

$$
f v_{p} d t=1 / 2 f v_{p} d t+1 / 2 \rho A_{c d t v}{ }_{p}^{2}
$$

d'où $\mathrm{f}=\rho \mathrm{Acv}$, équation connue comme exprimant l'écuilitbre local.

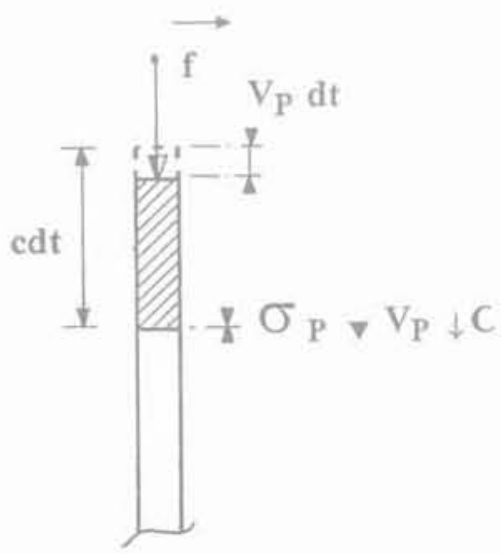

FG, 1 Onde de compression dans une tige. Stress wave into a drill-string. Cette relation s'écrit aussi $\mathrm{f}=\frac{E A}{\mathrm{c}} \mathrm{v}_{\mathrm{p}}$ ou $\mathrm{f}=\mathrm{Iv} \mathrm{v}_{\mathrm{p}}, \mathrm{I}$
étant l'impédance de la tige.

Si l'on raisonne en énergie, ou en puissance (dérivée de l'énergie par rapport au temps), on pourra dire que la puissance générée par le déplacement de la force, $\mathrm{fv}_{\mathrm{p}}$, se retrouve véhiculée par l'onde dans la tige, et qu'elle est proportionnelle au carré de la force: $P=\frac{c}{E A} f^{2}=\frac{f^{2}}{I}$. Elle ne dépend pas du signe de la force.

Pour compléter les notations que nous allons utiliser par la suite, schématisons un vibreur; ce dernier comporte (voir figure 2) :

- une masse a morte " $M_{0}$, qui ne participe pas à la vibration, mais exerce une forte constante $F_{0}=M_{0} g$ sur l'élément (la tige); il s'agit là d'une différence essentielle avec le battage ;

- deux masselottes de masse m chacune, symétriques, tournant en sens inverse avec une vitesse anqulaire $\omega$ qui génèrent donc chacune une force centrifuge $: 1 / 2 F_{c}=m^{2}(e=$ excentrement des masselottes $)$.

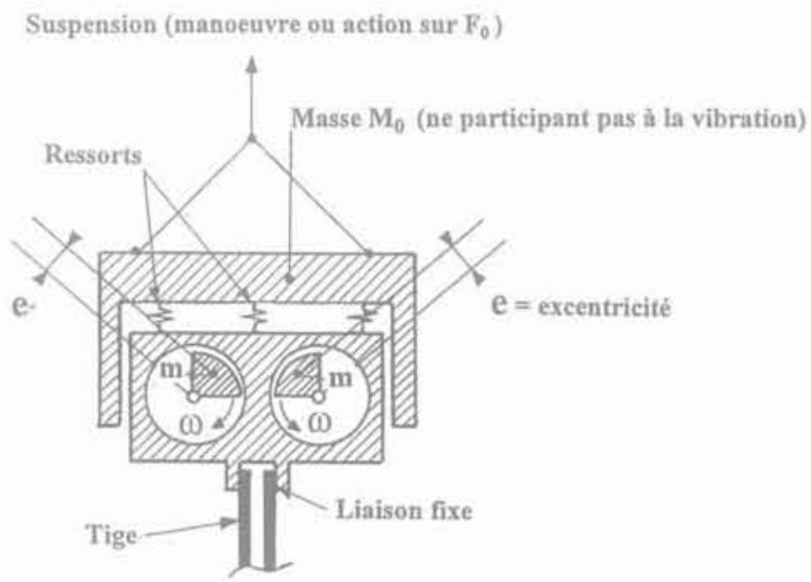

FiG.2 Représentation schématique d'un vibreur Pattern of vibratory device.

Les composantes horizontales s'annulent et les composantes verticales s'ajoutent pour donner une force verticale périodique sinusoïdale:

$$
f_{c}=F_{c} \cos \omega t=2 m \text { e } \omega^{2} \cos \omega t=M_{t} \omega^{2} \cos \omega t,
$$

$M_{+}$étant le moment du vibreur. Celui-ci étant liẻ rigidement à la tige, c'est donc cette force qui s'exerce sur le sommet de la tige.

\section{2}

\section{Amplitude du mouvement}

Les fabricants de vibreurs parlent souvent d'une "amplitude maximale théorique », suivant un raisonnement qui n'est pas toujours valide. C'est le déplacement vertical total du système vibrant au cours d'une rotation des excentriques ; si l'on appelle $m$ la masse totale qui doit être « excitée » par les masselottes, en supposant que cette masse se comporte de manière uniforme et rigide, on peut écrire : $\mathrm{f}_{\mathrm{c}}=\mathrm{m}_{\mathrm{v}} \gamma$

$$
\gamma=\frac{\mathrm{d}^{2} \mathrm{z}}{\mathrm{dt}^{2}} \quad \mathrm{z}=\text { déplacement vertical }
$$

$$
\frac{\mathrm{d}^{2} \mathrm{z}}{\mathrm{dt}^{2}}=\frac{\mathrm{f}_{\mathrm{c}}}{\mathrm{m}_{\mathrm{v}}}=\frac{\mathrm{F}_{\mathrm{c}} \cos \omega \mathrm{t}}{\mathrm{m}_{\mathrm{v}}}
$$




$$
\begin{gathered}
\frac{d z}{d t}=\frac{F_{c}}{m_{v}} \int_{0}^{t} \cos \omega d t=\frac{F_{c}}{\omega m_{v}} \sin \omega t \\
z=\frac{F_{c}}{\omega m_{v}} \int_{0}^{t} \sin \omega d t=\frac{F_{c}}{\omega^{2} m_{v}}(1-\cos \omega t)
\end{gathered}
$$

L'amplitude du mouvement est donc: $\frac{2 \mathrm{~F}_{\mathrm{c}}}{\omega^{2} \mathrm{~m}_{\mathrm{v}}}$ ou, si l'on fait intervenir le moment du vibreur $M_{3}$, $\frac{2 M_{t}}{m_{v}}$. En régime permanent, la puissance moyenne fournie est nulle dans cette hypothèse, car la tige restitue au vibreur l'énergie qu'elle reçoit de lui.

Nous avons montré, dans un article précédent, que pour une tige élastique de longueur finie L, soumise à une force instantanée $\mathrm{F}$ à une de ses extrémités, du fait des réflexions des ondes, les vitesses particulaires aux extrémités suivaient une progression arithmétique de raison $2 \mathrm{v}_{1}=2 \mathrm{~F} / \mathrm{I}$ avec un pas de temps égal à $2 \mathrm{~L} / \mathrm{c}$, l'accroissement moyen de vitesse par unité de temps étant égal à la force F divisée par la masse totale de la tige. Par conséquent, la valeur de l'amplitude que nous venons de calculer est celle du mouvement moyen stabilisé qui affecterait une tige élastique libre et de longueur finie, excitée par le vibreur considéré, à condition que la longueur de la tige soit faible au regard de la distance parcourue par l'onde pendant une période ( $2 \mathrm{~L} / \mathrm{c}$ petit devant $2 \pi / \omega$ ). Ce n'est pas vraiment le cas pour les vibreurs à fréquence standard utilisée pour enfoncer un profilé ou une palplanche :

$\mathrm{N}=1800$ tours $/$ minute $=30$ tours $/$ seconde

$$
\frac{2 \pi}{\omega}=\frac{1}{30} \text { de seconde }
$$

pour $\mathrm{L}=20 \mathrm{~m}$ et $\mathrm{c}=5000 \mathrm{~m} / \mathrm{s}$ (vitesse des ondes dans l'acier)

$$
\frac{2 L}{C}=\frac{40}{5000}=\frac{8}{1000} \text { de seconde, }
$$

soit 4 fois plus petit que $\frac{2 \pi}{\omega}$.

Si l'on veut étudier le mouvement généré par le vibreur solidaire d'une tige élastique de longueur finie et libre, on se trouve devant le même problème que celui des cordes vibrantes soumises à des vibrations forcées. Le profilé (la tige) n'est pas une boule de billard (corps rigide) mais un ressort (corps élastique). Le problème peut être modélisé à l'aide de masses rigides et de ressorts et traité numériquement. Mais on peut aussi l'aborder analytiquement (méthode des caractéristiques).

Raisonnons, pour simplifier, sur une tige de longueur infinie, de sorte que l'on ait qu'un seul train d'onde, sans réflexion. La vitesse particulaire au sommet sera

$$
v=\frac{f_{c}}{I}=\frac{F_{c}}{I} \cos \omega t=\frac{d z}{d t}
$$

d’où:

$$
z=\frac{F_{c}}{I} \int_{0}^{t} \cos \omega t d t=\frac{F_{c}}{l \omega} \sin \omega t
$$

L'amplitude du mouvement est $\frac{2 F_{c}}{I \omega}=\frac{2 M_{t} \omega}{I}$.

Elle est proportionnelle, pour un vibreur donné, à la vitesse angulaire et inversement proportionnelle à l'impédance de la tige; mais elle n'est pas nulle, comme le voudrait le premier calcul, puisque la masse d'une tige indéfinie est infinie!

Toute l'énergie fournie par le vibreur est envoyée dans la tige; la puissance moyenne consommée est:

$$
P=\frac{\omega}{2 \pi} \int_{0}^{\frac{2 \pi}{\omega}} \frac{f_{c}^{2}}{I} d t=\frac{F_{c}^{2}}{2 I}=\frac{M_{t}^{2} \omega^{4}}{2 I} \text {. La masse de tige }
$$

mise en mouvement croit linéairement avec le temps, puisque l'onde n'est pas réfléchie.

Cette puissance est proportionnelle à la puissance quatrième de la fréquence pour un vibreur ayant des masselottes et une excentricité données. Posons-nous maintenant la question de savoir comment cette énergie peut être utilisée pour la pénétration de la tige dans le sol.

\section{3}

\section{Amortissement et pénétration dans le sol}

La modélisation la plus simple consiste à considérer un milieu de comportement rigide plastique et un amortissement sous forme de forces résistantes de cisaillement le long de la tige en contact avec le sol proportionnelles à la vitesse particulaire. Le sol est alors représenté par deux paramètres:

- un coefficient $\mathrm{K}$, ayant les dimensions d'une contrainte par unité de vitesse. L'effet de l'amortissement est de faire diminuer l'intensité (la contrainte) d'une onde en fonction exponentielle de son parcours $\mathrm{z}$ :

$$
\sigma_{\mathrm{a}}=\sigma \exp \left[-\mathrm{Kp} \frac{|\mathrm{z}|}{\mathrm{I}}\right]
$$

I: impédance de la tige, $\mathrm{p}=$ périmétre en contact avec le sol:

- la résistance dynamique en pointe q. L'énergie véhiculée par l'onde qui n'a pas été dissipée dans son trajet jusqu'à la pointe est intégralement réfléchie sous la forme d'une onde de même polarité tant que la contrainte à l'extrémité reste inférieure à $q_{d}$; lorsque cette contrainte atteint $\mathrm{q}_{\text {, }}$, elle ne dépasse pas cette valeur et toute l'énergie excédentaire est utilisée pour la pénétration.

En tenant compte de ces hypothèses, nous pouvons déterminer la vitesse moyenne de pénétration de la tige dans un sol caractérisé par les paramètres $\mathrm{K}$ et $\mathrm{q}_{\mathrm{d}}$ pour l'onde incidente générée par le vibreur et transmise par la tige.

Rappelons les paramètres utilisés :

$\begin{array}{ll}\text { vibreur: } & \omega, F_{\sigma}, F_{c} \\ \text { tige (profilé) : } & \text { A, } \rho, E, p \\ \text { sol : } & K, q_{d}\end{array}$

soient 9 paramètres.

Tous ces paramètres sont indépendants, mais ils n'interviennent pas tous indépendamment dans le calcul, comme nous le verrons. 
Nous posons :

$\sigma_{0}=\frac{F_{0}}{A} \sigma_{c}=\frac{F_{c}}{A}$

$\sigma=\sigma_{c} \sin \omega t ;(t=$ temps $)$

$\sigma_{c a}=\sigma_{c} \exp \left[-\mathrm{Kp} \frac{|\mathrm{z}|}{\mathrm{I}}\right]$

$Q_{\mathrm{d}}=\mathrm{Aq}_{\mathrm{d}}$

Nous admettons que l'amortissement n'affecte que la contrainte variable et que $\sigma$ reste inférieur à $q_{d}$. En pointe, tant qu'elle $n^{\prime}$ a pas atteint $\mathrm{q}_{d}$, la contrainte s'exprime par: $\sigma_{\mathrm{p}}=\sigma_{\mathrm{o}}+2 \sigma_{\mathrm{c}}$ sin $\omega t$.

Lorsque cette contrainte atteint la valeur plafond $q_{4}$ elle ne la quitte plus et la vitesse particulaire en pointe

s'écrit: $v_{p}=\frac{C}{E}\left(\sigma_{0}+2 \sigma_{c a} \sin \omega t-q_{d}\right)$.

La pointe se déplace avec cette vitesse $v_{\mathrm{g}}$ pendant un temps allant de $t_{1}$ à $t_{2}$ tels que :

$\mathrm{q}_{\mathrm{d}}=\sigma_{0}+2 \sigma_{\mathrm{ca}} \sin \omega \mathrm{t}_{1}$

soit $t_{1}=\frac{1}{\omega} \arcsin \left[\frac{q_{d}-\sigma_{0}}{2 \sigma_{c a}}\right]\left(\arcsin <\frac{\pi}{2}\right)$

et $t_{2}$ tel que $\omega t_{2}=\pi$ - $\omega t_{1}$ (voir figure 3 ).

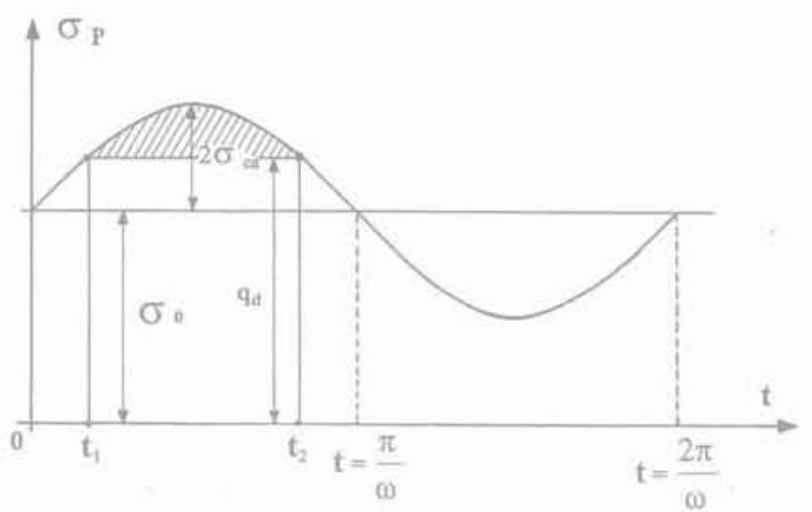

FG. 3 Contrainte en pied de tige. Toe stress.

Le déplacement $\delta$ de la pointe est donnée par:

$$
\delta=\int_{t_{1}}^{t_{2}} v_{p} d t=\frac{c}{E} \int_{t_{1}}^{t_{2}}\left(\sigma_{0}-q_{d}+2 \sigma_{\omega} \sin \omega\right) t d t
$$

au facteur $\frac{C}{E}$ près, $\delta$ est représenté par l'aire hachu-

rée de la figure 3 .

Tous calculs faits, il vient :

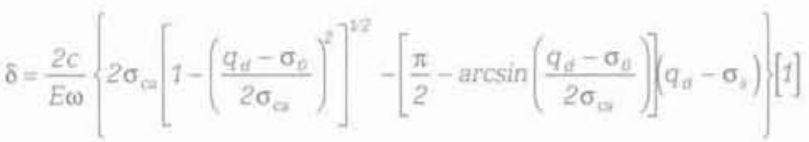

La vitesse moyenne de pénétration est égale à

$\delta \times \frac{\omega}{2 \pi}$.

$\mathrm{V}=\frac{\mathrm{c}}{\pi \mathrm{E}}\left\{2 \sigma_{\mathrm{ca}}\left[1-\left(\frac{\mathrm{q}_{\mathrm{d}}-\sigma_{0}}{2 \sigma_{c a}}\right)^{2}\right]^{1 / 2}-\left[\frac{\pi}{2}-\arcsin \left(\frac{\mathrm{q}_{d}-\sigma_{0}}{2 \sigma_{a}}\right)\right]\left(\mathrm{q}_{\mathrm{d}}-\sigma_{0}\right)\right\}[2]$

avec:

$$
\sigma_{\mathrm{ca}}=\sigma_{\mathrm{c}} \exp \left[-\mathrm{Kp} \frac{|\mathrm{z}|}{\mathrm{I}}\right][3]
$$

On peut remarquer qu'il n'y a plus, dans ces deux dernières formules, que 7 paramètres indépendants. La vitesse de rotation angulaire $\omega$ n'intervient pas directement dans l'expression de la vitesse moyenne de pénétration $V$ (mais c'est elle qui détermine la valeur de $\sigma$ ). La puissance utilisée pour la pénétration est égale à

$$
P=V \cdot Q_{d}
$$

Elle est nulle si $\sigma_{\mathrm{o}}+2 \sigma_{\mathrm{ca}}=\mathrm{q}_{\mathrm{d}}$ (ou $\mathrm{F}_{\mathrm{o}}+2 \mathrm{~F}_{\mathrm{ca}}=\mathrm{Q}_{\mathrm{d}}$ ).

Elle est maximale si $\mathrm{F}_{\mathrm{o}}=\mathrm{Q}_{\mathrm{d}}$ (valeur limite de $\mathrm{F}_{\mathrm{o}}$ d'après notre hypothèse) et alors égale à : $\frac{F_{c a} F_{0}}{I \pi}$.

La puissance perdue du fait de l'amortissement est égale à : $\frac{2 F_{c}^{2}}{I}\left[1-\exp \left(-2 K p \frac{|z|}{I}\right) \mid\right.$

\section{4}

\section{Et le vibrofonçage?}

L'effet des vibrations verticales transmises à l'élément (tube, profilé) que l'on veut enfoncer dans un terrain par vibrofonçage est de déstructurer le sol au contact de cet élément et de faire ainsí diminuer les u frottements » qui s'opposent à la pénétration; cela ne peut être obtenu, bien entendu, qu'au prix d'une certaine dissipation d'énergie. La vitesse de pénétration dépend de l'application d'une force verticale permanente, sous forme d'un poids mort ; cette force verticale se limite à quelques dizaines de kilonewtons (20 à $100)$ auxquels s'ajoute le poids de l'élément vibrofoncé, du même ordre de grandeur.

La force centrifuge verticale, elle, va de 400 à $2500 \mathrm{kN}$ pour les vibreurs standard. La force impulsionnelle utilisable pour la pénétration atteint un maximum pouvant aller de 800 à $5000 \mathrm{kN}$ (doublement par réflexion de l'intensité de l'onde). Cette force reste généralement inférieure aux forces impulsionnelles qui peuvent être obtenues par battage, qui ne sont limitées que par la résistance de la matière constitutive de l'élément battu.

Le vibrofonçage est donc moins bien adapté que le battage pour foncer des éléments importants dans des terrains offrant une forte résistance en pointe ; mais on ne voit pas pourquoi il ne permettrait pas de foncer des éléments de force portante en rapport avec les forces impulsionnelles développées à la mise en fiche, sans qu'il soit nécessaire de les mettre au refus par battage.

Par ailleurs, il me semble que le vibrofonçage peut 
permettre d'obtenir, par quelques tests simples en début de chantier, des indications intéressantes pour prévoir le déroulement des opérations et même apprécier certaines caractéristiques des sols traversés. En effet, pour déterminer l'énergie dissipée par frottement, il suffit d'ajuster la force constante pour obtenir une pénétration nulle (mesurer, par exemple, la force de suspension du vibreur et de son profilé) et de mesurer l'énergie consommée par le vibreur. Connaissant cette énergie, il suffit alors de reprendre la pénétration pour apprécier la résistance dynamique en pointe, si l'on admet que toute l'énergie consommée l'est pour vaincre les frottements et la résistance en pointe. Par ailleurs, si l'on admet qu'un sol, du point de vue de sa réaction au vibrofonçage, peut être caractérisé par deux paramètres seulement ( $\mathrm{K}$ et $\mathrm{q}_{\mathrm{d}}$ ), les quelques formulations explicitées ici peuvent peut-être permettre à l'aide de ces tests, de caractériser un site par des profils de $\mathrm{K}$ et $\mathrm{q}_{d}$; on pourrait même imaginer un * pénétromètre vibrofonceur ! ! Enfin, pourquoi ne pas essayer d'établir, suivant les sites, des corrélations avec des caractéristiques statiques, ce qui permettrait de contrôler directement la portance d'éléments vibrofoncés?

\section{Conclusion}

Je n'ai pas eu l'intention (ni la possibilité !) de traiter ici de manière complète le problème de la pénétration d'une tige élastique par vibrofonçage dans un milieu avec amortissement proportionnel à la vitesse particulaire et réaction rigide - plastique en pointe. C'est un problème purement mécanique que l'on sait traiter de manière numérique et que l'on peut résoudre avec des hypothèses plus complexes ou plus nuancées concernant la réaction du sol. Mais il n'est peut-être pas indiqué, pour aborder des problèmes pratiques, de faire intervenir des hypothèses trop complexes, mettant en jeu des paramètres difficiles à interpréter de manière physique et encore plus à mesurer. J'ai donc voulu plaider pour une manière à la fois pragmatique et raisonnée d'aborder une recherche appliquée sur la bonne utilisation du vibrofonçage pour ficher dans le sol des éléments linéaires, portants ou non.

Cela en vaut certainement la peine, tant le vibrofonçage est largement utilisé dans les travaux publics.

\section{$\overline{\text { Bibliographie }}$}

Barré de Saint-Venant B. - - Mémoire sur le choc longitudinal de deux barres élas. tiques y. Journal de Mathématiques, 2 ser. XIII 1867, p. 237-376.

Gonin $\mathrm{H}$ - "Etude theerique du battage des corps élastiques élancés $x$. Annales ITBTP, Série Sols et Fondations, $n^{\circ} 155$ 1978.
Gonin H. - "Réflexions sur le battage des pieux 1. Revue Française de Géotechnique, $n^{\circ}$ 9, 1979.

Gonin $H_{\text {. }}$ - "Du pénétrométre dynamique au battage des pieux v. Revue Française de Géotechnique, n" 76, 1996.
Heritier B., Paquet J. - " Détermination de la capacitè portante des pieux par les

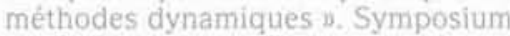
International de San Francisco, vol. 1. août 1985. 